\title{
Decrease in longitudinal strain in heart transplant recipients is associated with rejection
}

\author{
Helena Podrouzkovaa , Helena Bedanova ${ }^{b}$, Martin Tretina ${ }^{b}, J^{a}$ sef Korinek ${ }^{a, c}$, Radka Stepanovad, Jana Hruskova ${ }^{a}$, Petr Nemec ${ }^{b}$, \\ Tomas Konecny ${ }^{\mathrm{a}}$, Marek Orban ${ }^{\mathrm{a}, \mathrm{b}}$
}

Background. Around 20-40\% of heart transplant patients experience moderate to severe rejection within the first year after heart transplantation. Endomyocardial biopsy (EMB) is a gold standard for diagnosing heart transplant rejection. There is a need for non-invasive alternatives that allow for early, safe and reliable diagnosis of acute graft rejection prior to the onset of clinical symptoms.

Aims. Our aim was to investigate the potential of speckle tracking derived strain analysis in the diagnosis of acute graft rejection.

Methods. Patients indicated for EMB consented to a trans-thoracic echocardiography examination (TTE) within 2 hours of the EMB. Of this cohort, those with at least 2 EMBs separated $\geq 1$ week, and whose TTE could be analyzed for strain, were included. The relationship between strain and EMB results was evaluated.

Results. Of the 43 patients included (mean age $51.33 \pm 1.79,67 \%$ male), 23 had findings of rejection identified on at least one EMB and at least one EMB without rejection for comparison. A significant deterioration in the longitudinal strain during rejection compared to non-rejection was found on apical 4-chamber views $(-11.51 \pm 0.91$ vs $-13.48 \pm 0.96$, $P=0.025)$ and apical 2 -chamber views $(-11.84 \pm 0.78$ vs $-14.43 \pm 0.83, P=0.002)$. In the patients in whom no rejection was identified on either EBM, there was no significant change in longitudinal strain values at two different time points.

Conclusion. Worsening of longitudinal strain was associated with acute cellular rejection. Routine TTE-based strain analysis could help in early detection of cardiac rejection and timing of EMB.

Key words: echocardiography, heart transplantation, graft rejection

Received: August 4, 2014; Accepted with revision: April 14, 2015; Available online: April 29, 2015

http://dx.doi.org/10.5507/bp.2015.020

a ICRC, St. Anne's University Hospital in Brno, Czech Republic

${ }^{b}$ Center of Cardiovascular and Transplant Surgery, St. Anne's University Hospital in Brno

${ }^{c} 2^{\text {nd }}$ Department of Internal Medicine - Cardiology and Angiology, General University Hospital and $1^{\text {st }}$ Faculty of Medicine, Charles University in Prague

${ }^{d}$ Department of Biostatistics, ICRC, St. Anne's University Hospital in Brno

Corresponding author: Marek Orban, e-mail: maor@post.cz

\section{INTRODUCTION}

In the first year after heart transplantation, approximately $20 \%$ to $40 \%$ of patients experience moderate to severe, acute cellular rejection which remains one of the leading causes of death ${ }^{1}$. Endomyocardial biopsy (EMB) serves as a gold standard in diagnosing graft rejection, but its invasive nature makes it prone to associated complications ${ }^{2,3,4}$. Therefore, there is a need for a non-invasive diagnostic tool, which would allow early, safe and reliable diagnosis of acute graft rejection prior to onset of clinical symptoms.

Echocardiography has been tested as a promising non-invasive technique for detection of acute cellular graft rejection in early stages, but none of the conventional parameters were found reliable enough to replace regular EMB $\left(\right.$ ref. $\left.^{5}\right)$. Left ventricular (LV) ejection fraction (LV EF) is a traditionally used marker of systolic performance based on volume analyses and thus may not reveal subtle changes in contractility. Strain analysis is designed to quantify ventricular deformation in longitudinal, circumferential and radial directions on both segmental and global levels. Strain has shown to be more sensitive to early LV changes than LV EF in various pathologies ${ }^{6}$, and it has proven superior in prognosis prediction ${ }^{7,8}$. In the field of heart transplantation, the speckle tracking derived strain has been recently applied, normal values for clinically stable heart transplant recipients were established ${ }^{9}$, and an association between impaired strain and acute rejection was demonstrated in both animal ${ }^{10,11}$ and human studies ${ }^{12-14}$.

The aim of our study was to investigate the utility of speckle tracking derived strain analysis in the diagnosis of acute graft rejection in heart transplant recipients with preserved ejection fraction, and in detecting early subclinical phases of acute rejection.

\section{METHODS}

\section{Study population}

Study was designed as a single center prospective study. All consecutive heart transplant patients referred to our center for routine EMB between August 2008 
and September 2010 were eligible for recruitment to this study. Exclusion criteria were: 1) LV EF <55\%; 2) moderate or higher grade valvular pathology; 3 ) clinically significant pericardial effusion; 4) inadequate acoustic window; 5) moderate or higher grade cardiac allograft vasculopathy ${ }^{15}$ assessed by routine coronary angiography (performed one month and one year after the heart transplantation); 6) unwillingness of the patient to participate in this study; 7) unavailability of study personnel.

\section{Study protocol}

Our standard protocol post heart transplantation indicates EMB to be performed weekly during the first month after heart transplantation, every two weeks during the second and third months after heart transplantation, monthly during the fourth to sixth months, every three months until the end of the first year, and annually after that. The patients included in this study were thus examined at different time points after heart transplantation. Based on the EMB results they were divided into two groups: The "Rejection Group" consisted of patients who had at least one EMB diagnosed as rejection (grade 1B and worse), and at least one EMB diagnosed as no rejection" (grade 0 or 1A). These biopsies had to be temporally separated by $\geq 1$ week. The "Control Group" included those patients who did not have rejection on any of their EMB (patients with EMB results of grade 0 or $1 \mathrm{~A}$ ) during the study period. In case there was more than one EMB available with identical rejection grade in one individual, the examination with the best image quality was chosen for the analysis.

All patients involved in the study gave written informed consent. The study was in compliance with the declaration of Helsinki and was approved by institutional ethics committee.

\section{Endomyocardial biopsy}

EMB from different regions of right ventricle was performed using a jugular or trans-femoral approach. Biopsy findings were evaluated according to The International Society for Heart and Lung Transplantation guidelines from 1990 (ref. ${ }^{16}$ ) which were being routinely used in our institution at the time of the study.

\section{Echocardiography examination}

All patients underwent echocardiography within 2 hours of the EMB. Echocardiography images were obtained with ultrasound machine VIVID 7 (GE, Milwaukee, WI, USA). Routine echocardiography examination including chamber quantification was performed and evaluated according to the current guidelines ${ }^{17}$. The ejection fraction was calculated with the biplane Simpson's rule. Trans-aortic flow was recorded by pulsed wave Doppler. 2D loops of parasternal short axis view on the level of papillary muscles and apical four- and two-chamber views were digitally stored. For this purpose we used the highest possible frame rates (between 55 and 90 frames/s). Global circumferential and longitudinal strain and strain rate values were determined offline (Echo Pac system, General Electric, Inc.).

\section{Strain analysis}

Detailed description of strain analysis has been published previously ${ }^{18}$. Loops with visually assessed suboptimal tracking quality or loops, where the speckle tracking could not be obtained for at least 4 of the 6 segments were not included in the analysis. The strain was analyzed by an investigator blinded to the biopsy results. Strain was assessed in three consecutive heart cycles and an average of these values was used for analysis. The assessed strain parameters were longitudinal peak systolic strain and strain rate in apical 2- and 4-chamber view and circumferential peak systolic strain and strain rate.

The reproducibility of strain and strain rate measurements were assessed in 10 randomly chosen patients. For intra-observer reproducibility the second analysis was performed three months after the first one. Inter-observer variability was evaluated by two independent observers H. P. and M. O.

\section{Statistical analysis}

All continuous data were expressed as mean and standard error of the mean, if not stated otherwise. The categorical data was shown as number and percentage. Most

Table 1. Baseline characteristics of both groups, patients who experienced rejection episode and patients who were free from rejection - controls.

\begin{tabular}{lccc}
\hline & $\begin{array}{c}\text { Patients with rejection episode } \\
\text { during follow up }(\mathrm{n}=23)\end{array}$ & $\begin{array}{c}\text { Patients without rejection episode } \\
\text { during follow up- controls }(\mathrm{n}=20)\end{array}$ & $P$ \\
\hline Male gender & $16(70 \%)$ & $13(65 \%)$ & 0.750 \\
Recipient age & $53.78 \pm 1.83$ & $48.50 \pm 3.16$ & 0.364 \\
Donor age & $36.91 \pm 2.51$ & $38.50 \pm 3.26$ & 0.709 \\
BMI $\left(\mathrm{kg} / \mathrm{m}^{2}\right)$ & $25.69 \pm 0.53$ & $25.35 \pm 1.06$ & 0.914 \\
Diabetes mellitus & $16(70 \%)$ & $11(55 \%)$ & 0.324 \\
Hypertension & $16(70 \%)$ & $7(35 \%)$ & 0.023 \\
Hyperlipidaemia & $17(74 \%)$ & $14(70 \%)$ & 0.775 \\
Ischemic time (min) & $161.87 \pm 52.84$ & $142.95 \pm 48.30$ & 0.697 \\
\hline
\end{tabular}

$\mathrm{BMI}=$ body mass index of the recipient in the time of transplantation

Data are displayed as either number and percentage in categorical variables or mean \pm standard error of the mean in continuous variables. 
variables did not present normal distribution, therefore non-parametric tests were applied. The difference between two continuous variables for paired data was analyzed using Wilcoxon Matched Pairs test for dependent samples. For independent samples Mann-Whitney U test was used. To compare the differences in categorical variables, the $\chi^{2}$ test was applied. If a number smaller than 5 occurred in a cell of a contingency table, the Yates correction for $\chi^{2}$ test was used. All analyses were carried out using a statistical program Statistica, StatSoft. Inc.

\section{RESULTS}

\section{Clinical data}

Forty-three patients after heart transplantation were enrolled in the study. All included patients were in sinus rhythm without cardiac pacing. Heart transplantation in all patients was performed in a bi-atrial approach. All patients received standard immunosuppressive therapy per local protocol (either a combination of cyclosporin, mycophenolate mofetil and prednisone, or tacrolimus, mycophenolate mofetil and prednisone). Patients who presented both with and without rejection during the course of the study $(n=23)$ were included in the Rejection Group. Of the patients without rejection, 20 were included in the Control group and underwent complete analysis. Baseline characteristics of the two groups are shown in Table 1. All included patients were Caucasian, and the most frequent cause of heart failure leading to heart transplantation was dilated cardiomyopathy. All included patients were in the range between 11 days to 4 years after heart transplantation. In the Rejection Group, most patients presented with rejection grade $1 \mathrm{~B}$; there were only 7 patients in rejection grade 2 and one in $3 \mathrm{~A}$ and one in $3 \mathrm{~B}$. Coronary angiography performed one month after heart transplantation revealed mild cardiac allograft vasculopathy ${ }^{15}$ in three patients, all of whom were in the rejection group. The rest of the patients were free from cardiac allograft vasculopathy.

\section{Echocardiographic data}

The analyses of echocardiography data are presented in detail in Table 2 and Fig. 1-3. The paired data analysis revealed statistically significant deterioration of longitudinal strain in both apical 4-and 2 - chamber view as well as worsening of longitudinal systolic strain rate in apical 2 chamber view during rejection compared to non-rejection. Neither the change in circumferential strain and strain rate, nor the change in longitudinal strain rate in apical 4- chamber view had any statistically significant relationship to the presence of rejection. Due to the small number of rejection grades $2,3 \mathrm{~A}$ and $3 \mathrm{~B}$, it was not possible to find a significant correlation between the magnitude of

Table 2. Global strain and strain rate values and echocardiography characteristics of both the Rejection group and the Control group.

\begin{tabular}{|c|c|c|c|c|c|c|}
\hline & \multicolumn{2}{|c|}{ Rejection group $(\mathrm{n}=23)$} & \multirow{2}{*}{$P$} & \multicolumn{2}{|c|}{ Control group $(n=20)$} & \\
\hline & AR- & $\mathrm{AR}^{+}$ & & $\mathrm{T} 1$ & $\mathrm{~T} 2$ & \\
\hline ongitudinal strain 2-chamber view (\%) & $-14.43 \pm 0.83$ & $-11.84 \pm 0.78$ & 0.002 & $-14.41 \pm 1.72$ & $-14.06 \pm 1.78$ & 0.50 \\
\hline $\begin{array}{l}\text { Longitudinal peak systolic strain rate } \\
\text { 2-chamber view }\left(\mathrm{s}^{-1}\right)\end{array}$ & $-0.99 \pm 0.07$ & $-0.80 \pm 0.06$ & 0.006 & $-0.87 \pm 0.13$ & $-0.60 \pm 0.32$ & 0.114 \\
\hline ongitudinal strain 4- chamber view (\%) & $-13.48 \pm($ & $-11.51 \pm 0.91$ & 25 & -14.76 & -14.06 & 0.730 \\
\hline ) & -0.78 & -0.7 & 0.422 & -0.86 & .05 & .850 \\
\hline strain $(\%)$ & -18.83 & -18.11 & 0.619 & -20.4 & -18.6 & 0.004 \\
\hline $\begin{array}{l}\text { ircumferential peak systolic strain rate } \\
\mathrm{s}^{-1} \text { ) }\end{array}$ & -1.39 & & 0.619 & -1.3 & 0.20 & 0.048 \\
\hline$V$ mass & 213.8 & 226.5 & 0.330 & 209.1 & 2.62 & 0.627 \\
\hline $\mathrm{CV} n$ & 109.81 & 116. & 0.303 & 107.5 & 5.53 & 0.709 \\
\hline nd-d & $89.22 \pm 6.15$ & $98.22 \pm 7.28$ & 0.088 & $97.85 \pm 5.63$ & $98.55 \pm 6.99$ & 0.970 \\
\hline nd- & $45.47 \pm 2.82$ & $50.17 \pm 3.55$ & 0.094 & $50.43 \pm$ & $50.53 \pm 2.82$ & 0.940 \\
\hline ind-sy & $34.22 \pm 2.56$ & $39.22 \pm 3.17$ & 0.064 & $38.50 \pm 2.68$ & $37.95 \pm 3.06$ & 0.852 \\
\hline End-systolic volume index ${ }^{a}$ & $17.46 \pm 1.22$ & $20.06 \pm 1.60$ & 0.077 & $19.82 \pm 1.20$ & $19.46 \pm 1.29$ & 0.823 \\
\hline LEF & $61.70 \pm 1.10$ & $60.39 \pm 0.85$ & 0.202 & $60.90 \pm 1.14$ & $61.75 \pm 0.91$ & 0.401 \\
\hline Septal & $13.52 \pm 0.62$ & $13.39 \pm 0.60$ & 0.629 & $13.85 \pm 0.61$ & $13.60 \pm 0.63$ & 0.551 \\
\hline Post & $12.74 \pm 0.48$ & $12.96 \pm 0.42$ & 0.570 & $12,15 \pm 0.41$ & $11.30 \pm 0.40$ & 0.083 \\
\hline Time after heart transplantation (days) & $351 \pm 86$ & $385 \pm 93$ & 0.401 & $88 \pm 22$ & $253 \pm 54$ & \\
\hline
\end{tabular}

a indexed to body surface area of the recipient

The data from the patient in Rejection group were acquired at different clinical scenarios; as either no-rejection values (AR-) or values in the same patient in rejection (AR+). The data from the patients in Control group (subjects who did not experience rejection) were acquired at different time points T1 and T2. Values are presented as mean \pm standard error of the mean. $P$-values are displayed for Wilcoxon matched-pairs test, $P$-values lower than 0.05 are highlighted. 


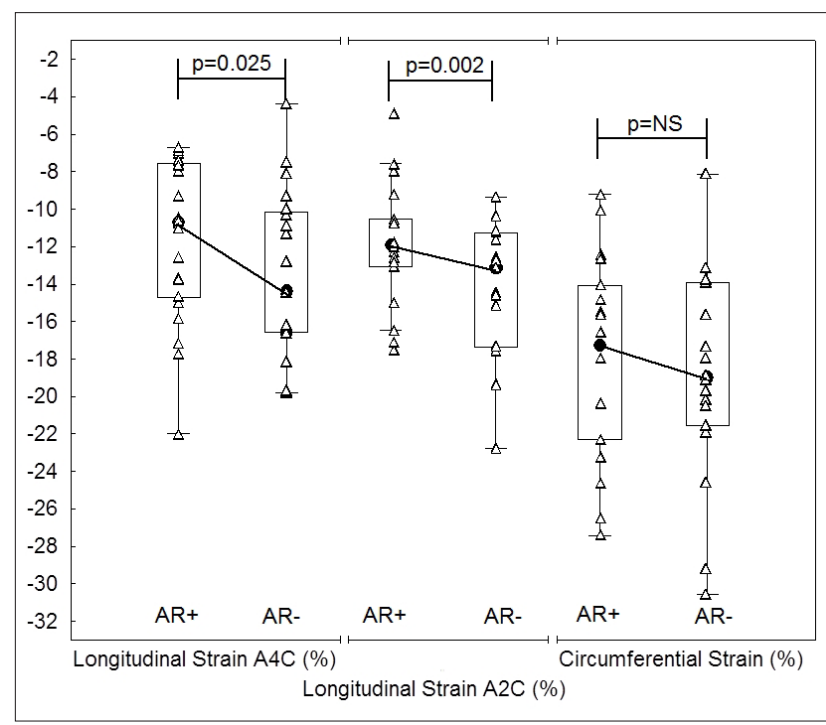

Fig. 1. Boxplots of individual strains of patients in the Rejection Group, where the triangles stand for the raw data, the connecting line points to the median, box stands for quartiles and the whiskers for non -outlier range. Graphs show the differences in individual strains in the time of rejection $(\mathrm{AR}+)$ to the time when patients were rejection free (AR-). Even though these are paired data, and $P$-values show the result of paired data analysis, boxplots were used to provide a better overview.

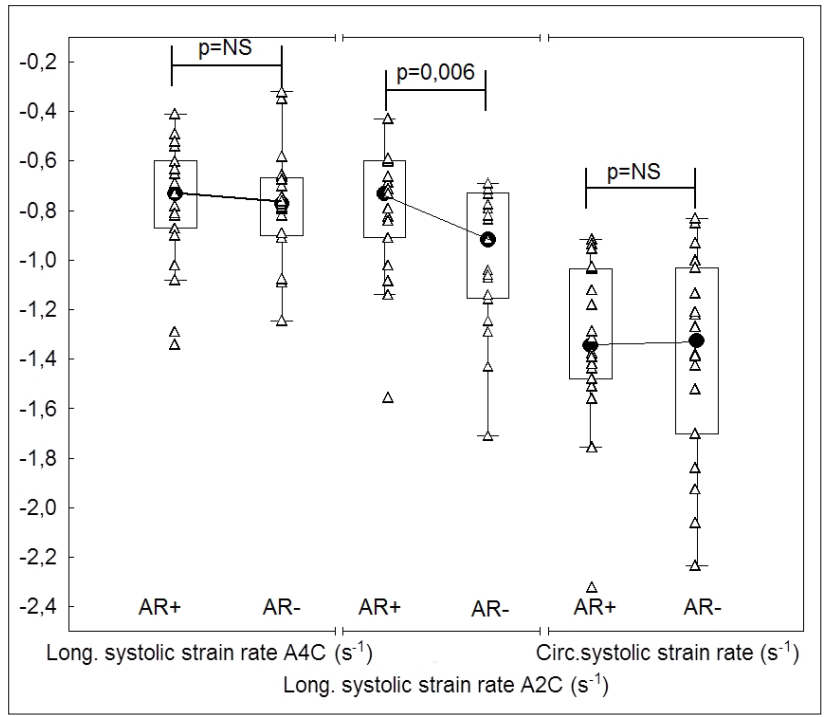

Fig. 2. Boxplots of individual strain rates with the same details as described in fig. 1.

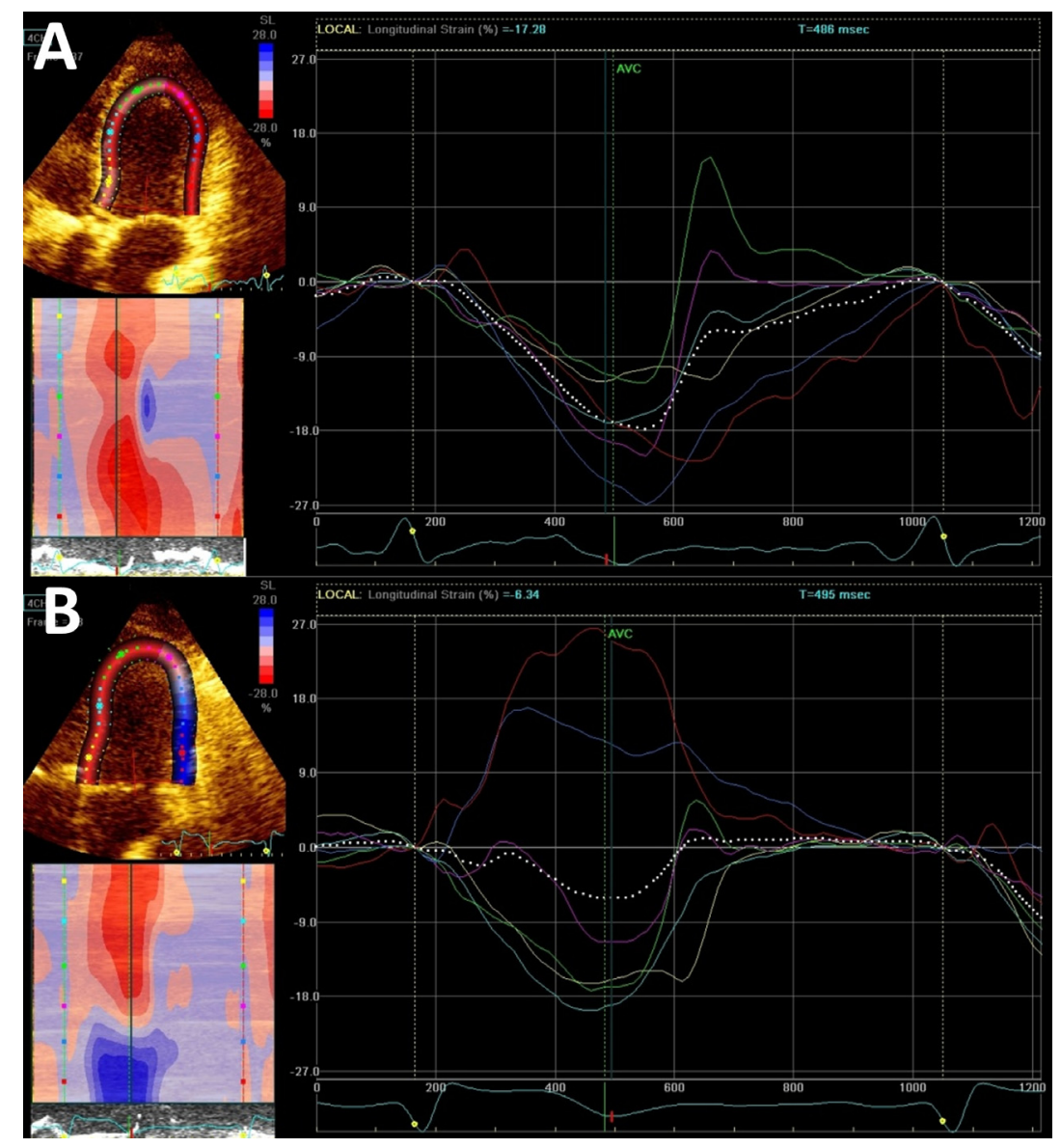

Fig. 3A. Speckle tracking derived longitudinal strain curve in heart transplant patient when free from rejection. The colored curves represent strains of individual segments and the white dotted line stands for global strain, i.e. mean for all segments.

Fig. 3B. Shows the same patient as in figure 3A, this time in rejection. Obvious decrease in absolute values of global systolic strain can be attributed to local dyssynchrony in lateral segments in this patient. Nevertheless, no regularity was observed in the rejection strain pattern. 
strain deterioration and severity of rejection. No statistically significant changes in longitudinal strain or strain rate were found in the control group. However, there was a significant worsening in circumferential strain and strain rate over time in the control group.

Intra-observer variation for peak systolic strain and peak systolic strain rate were $2.26 \pm 1.9 \%$ and $0.97 \pm 3.0 \%$ respectively. The relative difference in inter-observer measurements for peak systolic strain and peak systolic strain rate were $4.4 \pm 2.4 \%$ and $3.35 \pm 3.1 \%$ respectively.

There was no statistically significant difference between the groups of patients with and without rejection episode during follow up in terms of LV mass index, LV end-diastolic volume index, LV end-systolic volume index, LV ejection fraction, septal or posterior wall thickness or E/e'. Similarly, there was no significant change in these variables in the paired data analyses either in the group of patients with or without rejection episode during follow up (Table 2). No significant progression of LV hypertrophy was observed in either group.

\section{DISCUSSION}

In this prospective, single-center study, we found evidence of rejection on EMB to be related to the worsening of longitudinal strain in both apical 2- and 4-chamber. This is the first clinical study showing the utility of speckle tracking to evaluate acute cellular rejection in patients after heart transplantation in a paired data setting and where the echocardiography was performed within 2 hours after the EMB.

Acute rejection was considered as $1 \mathrm{~B}$ or worse according to the older classification from 1990 (ref. ${ }^{16}$ ). This older classification, compared to the new one ${ }^{19}$, allows us to differentiate between mild grades of rejection, which was appropriate in our study given our goal to evaluate the sub-clinical early stages of rejection.

Our findings are in concordance with most of previously published animal ${ }^{10,11}$ and human ${ }^{12,13}$ data. The heart graft rejection is characterized by immune cell infiltration, edema and myocyte damage, which results in impaired mechanical LV function, and decrease in strain. Additionally, global longitudinal strain seems to be parameter sensitive enough to reveal a chronic low degree rejection often undetectable by biopsy, which may be responsible for poor prognosis in heart transplant recipients ${ }^{20,21}$.

The circumferential strain in our study showed no relation to rejection episodes, however a trend toward decrease over time after heart transplantation was observed.

Our baseline (non-rejection) systolic longitudinal strain values are in agreement with those previously published ${ }^{22,23,9}$. In all above mentioned studies, as well as in our study, the same software was used with a 16 segmental model of the LV making data comparison between studies reasonable.

In animal studies, very good correlations of strain values to rejection grades have been found. Additionally, strain improved in reaction to immunosuppression treatment. ${ }^{10}$ Nevertheless, there are a few distinctions between animal and human studies. The rats in heart transplantation studies received no immunosuppressive treatment at all, so all of them soon developed high rejection grades. The baseline values were acquired from isografts, where the transplanted heart is genetically identical to the recipient. In these studies, often a heterotopic transplant model of a non - working heart was applied ${ }^{10}$, where the transplanted heart lacks physiological loading. This may also influence contractility and immune cell infiltration in the heart.

Strain decrease is a non-specific finding, which limits its potential clinical application. Besides the rejection and cardiac allograft vasculopathy ${ }^{23}$, there are other factors potentially influencing the LV contractility, such as hypertension, diabetes mellitus, hyperhomocysteinaemia, pulmonary hypertension, LV preload, LV hypertrophy, renal failure, advancing age and post-transplantation infections. In early post-transplantation period, the LV strain is influenced by time of ischemia during operation, reperfusion injury and denervation. Donor variables that may also influence LV performance, are donor age, LV hypertrophy, and gender mismatch ${ }^{1}$. A potential clinical application of strain measurements, rather than diagnosing rejection as such, could be an improvement in timing of the EMB. Deterioration of the baseline values acquired in non-rejection would be an indication to perform EMB. Thus, we could reduce the number of biopsies, if this approach was approved in future multi-center studies with unified methodology.

There are several limitations to this study. The small number of patients who presented both with and without acute rejection (rejection group) was expected and is a common feature of the majority of studies in the transplantation field. A potential limitation to our study is higher number of patients with hypertension in the group of patients with rejection episode in the follow up comparing to controls. The higher prevalence of hypertension could have contributed to impaired strain in this group. The different timing of rejection episodes after heart transplantation is another limitation. Some patients experienced rejection before and some after baseline (non-rejection) data acquisition. If longitudinal strain values were changing over time, this might have affected the results. However, there are studies showing, that longitudinal strain values in clinically stable heart transplant recipients remain attenuated at all time points compared to healthy population and do not change even in a two ${ }^{20}$ or three year follow up ${ }^{23}$. Thus, different timing of episodes should not significantly bias our data.

\section{CONCLUSION}

Using paired-data analyses, we showed that worsening of longitudinal systolic strain in heart transplant patients may be associated with acute cellular rejection, revealing milder, sub-clinical grades. This could be of major clinical relevance if these findings are confirmed in a larger study. Due to small number of higher rejection grades in this study, we could not assess the correlation between strain values and individual rejection grades. 


\section{ABBREVIATIONS}

A2C, Apical two-chamber view; A4C, Apical fourchamber view; AR, Episodes without rejection; AR+, Episodes with rejection; BMI, Body mass index; EMB, Endomyocardial biopsy; LV, Left ventricle; LVEF, Left ventricle ejection fraction.

Acknowledgement: This study was supported in part by the European Regional Development Fund - Project FNUSA-ICRC (No. CZ.1.05/1.1.00/02.0123) and in part by Internal Grant Agency of Ministry of Healthcare of the Czech Republic (IGA MZ NS 10627-3/2009). JK was also supported by PRVOUK-P35/LF1/5 and project from OP Prague Competitiveness, reg.no. CZ.2.16/3.1.00/21565.

Author contributions: HP, MO: literature search; MO, JK: study design; HB, MO, MT: data acquisition; HP: echodata analysis; RS, JH: statistical analyses; HP: interpretation of the results, manuscript writing; MO, HB, MT, JK, PN, TK manuscript revision; All authors: final approval. Conflict of interest statement: The authors state that there are no conflicts of interest regarding the publication of this article.

\section{REFERENCES}

1. Stehlik J, Edwards LB, Kucheryavaya AY, Benden C, Christie JD Dobbels F, Kirk R, Rahmel AO, Hertz MI. The Registry of the International Society for Heart and Lung Transplantation: Twentyeighth Adult Heart Transplant Report 2011. J Heart Lung Transplant 2011;30:1078-94.

2. Holzmann M, Nicko A, Kühl U, Noutsias M, Poller W, Hoffmann W, Morguet A, Witzenbichler B, Tschöpe C, Schultheiss H-P, Pauschinger M. Complication rate of right ventricular endomyocardial biopsy via the femoral approach: a retrospective and prospective study analyzing 3048 diagnostic procedures over an 11-year period. Circulation 2008;118:1722-8.

3. Saraiva F, Matos V, Gonçalves L, Antunes M, Providência L a Complications of endomyocardial biopsy in heart transplant patients: a retrospective study of 2117 consecutive procedures. Transplant Proc 2011;43:1908-12.

4. Yilmaz A, Kindermann I, Kindermann M, Mahfoud F, Ukena C, Athanasiadis A, Hill S, Mahrholdt H, Voehringer M, Schieber M Klingel K, Kandolf R, Böhm M, Sechtem U. Comparative evaluation of left and right ventricular endomyocardial biopsy: differences in complication rate and diagnostic performance. Circulation 2010;122:900-9.

5. Estep JD, Shah DJ, Nagueh SF, Mahmarian JJ, Torre-Amione G, Zoghbi W a. The role of multimodality cardiac imaging in the transplanted heart. JACC Cardiovasc Imaging 2009;2:1126-40.

6. Gorcsan J, Tanaka H. Echocardiographic assessment of myocardial strain. J Am Coll Cardiol 2011;58:1401-13.

7. Antoni ML, Mollema S, Delgado V, Atary JZ, Borleffs CJW, Boersma E, Holman ER, van der Wall EE, Schalij MJ, Bax JJ. Prognostic importance of strain and strain rate after acute myocardial infarction. Eur Heart J 2010;31:1640-7.

8. Stanton T, Leano R, Marwick TH. Prediction of all-cause mortality from global longitudinal speckle strain: comparison with ejection fraction and wall motion scoring. Circ Cardiovasc Imaging 2009;2:356-64

9. Syeda B, Höfer P, Pichler P, Vertesich M, Bergler-Klein J, Roedler S, Mahr S, Goliasch G, Zuckermann A, BinderT. Two-dimensional speckle-tracking strain echocardiography in long-term heart transplant patients: a study comparing deformation parameters and ejection fraction derived from echocardiography and multislice computed tomography. Eur J Echocardiogr 2011;12:490-6.

10. Pieper GM, Shah A, Harmann L, Cooley BC, lonova IA, Migrino RQ. Speckle-tracking 2-dimensional strain echocardiography: a new noninvasive imaging tool to evaluate acute rejection in cardiac transplantation. J Heart Lung Transplant 2010;29:1039-46.

11. Wu YL, Ye Q, Ho C. Cellular and Functional Imaging of Cardiac Transplant Rejection. Curr Cardiovasc Imaging Rep 2011;4:50-62.

12. Kato TS, Oda N, Hashimura K, Hashimoto S, Nakatani T, Ueda H-I, Shishido T, Komamura K. Strain rate imaging would predict sub-clinical acute rejection in heart transplant recipients. Eur J Cardiothorac Surg 2010;37:1104-10.

13. Marciniak A, Eroglu E, Marciniak M, Sirbu C, Herbots L, Droogne W, Claus P, D'hooge J, Bijnens B, Vanhaecke J, Sutherland GR. The potential clinical role of ultrasonic strain and strain rate imaging in diagnosing acute rejection after heart transplantation. Eur J Echocardiogr 2007;8:213-21.

14. Lisi M, Cameli M, Tacchini D, Ballo P, Maccherini M, Mondillo S. Two-dimensional speckle tracking echocardiography of acute cardiac transplant rejection following pregnancy. J Clin Ultrasound 2012;40:451-4.

15. Mehra MR, Crespo-Leiro MG, Dipchand A, Ensminger SM, Hiemann NE, Kobashigawa J a, Madsen J, Parameshwar J, Starling RC, Uber P a. International Society for Heart and Lung Transplantation working formulation of a standardized nomenclature for cardiac allograft vasculopathy-2010. J Heart Lung Transplant 2010;29:717-27.

16. Billingham ME, Cary NR, Hammond ME, Kemnitz J, Marboe C, McCallister HA, Snovar DC, Winters GL, Zerbe A. A working formulation for the standardization of nomenclature in the diagnosis of heart and lung rejection: Heart Rejection Study Group. The International Society for Heart Transplantation. J Hear Transpl 1990;9:587-93.

17. Lang RM, Bierig M, Devereux RB, Flachskampf F a, Foster E, Pellikka P a, Picard MH, Roman MJ, Seward J, Shanewise JS, Solomon SD, Spencer KT, Sutton MSJ, Stewart WJ. Recommendations for chamber quantification: a report from the American Society of Echocardiography's Guidelines and Standards Committee and the Chamber Quantification Writing Group, developed in conjunction with the European Association of Echocardiograph. J Am Soc Echocardiogr 2005;18:1440-63.

18. Meluzín J, Podroužková H, Pešl M, Leinveber P, Orban M. Speckle tracking echocardiography - a new tool for quantification of myocardial function. Cor Vasa 2008;50:28-33.

19. Stewart S, Winters GL, Fishbein MC, Tazelaar HD, Kobashigawa J, Abrams J, Andersen CB, Angelini A, Berry GJ, Burke MM, Demetris AJ, Hammond E, Itescu S, Marboe CC, McManus B, Reed EF Reinsmoen NL, Rodriguez ER, Rose AG, Rose M, Suciu-Focia N, Zeevi A, Billingham ME. Revision of the 1990 working formulation for the standardization of nomenclature in the diagnosis of heart rejection. J Heart Lung Transplant 2005;24:1710-20.

20. Eleid MF, Caracciolo G, Cho EJ, Scott RL, Steidley DE, Wilansky S Arabia F a, Khandheria BK, Sengupta PP. Natural history of left ventricular mechanics in transplanted hearts: relationships with clinical variables and genetic expression profiles of allograft rejection. JACC Cardiovasc Imaging 2010;3:989-1000.

21. Sarvari SI, Gjesdal O, Gude E, Arora S, Andreassen AK, Gullestad L, Geiran O, Edvardsen T. Early postoperative left ventricular function by echocardiographic strain is a predictor of 1-year mortality in heart transplant recipients. J Am Soc Echocardiogr 2012;25:1007-14.

22. Saleh HK, Villarraga HR, Kane GC, Pereira NL, Raichlin E, Yu Y, Koshino Y, Kushwaha SS, Miller FA, Oh JK, Pellikka PA. Normal left ventricular mechanical function and synchrony values by speckle-tracking echocardiography in the transplanted heart with normal ejection fraction. J Heart Lung Transplant 2011;30:652-8.

23. Pichler $P$, Binder $T$, Höfer $P$, Bergler-Klein J, Goliasch G, Lajic N, Aliabadi A, Zuckermann A, Syeda B. Two-dimensional speckle tracking echocardiography in heart transplant patients: three-year follow-up of deformation parameters and ejection fraction derived from transthoracic echocardiography. Eur Heart J Cardiovasc Imaging 2012;13:181-6. 